\title{
SPECTROSCOPIC MEASUREMENT AND ANALYSIS OF FAT IN MiLK
}

\author{
Hana Vaskova, Martina Buckova \\ Tomas Bata University in Zlin, Faculty of Applied Informatics, Nad Stranemi 4511, Zlin 760 05, Czech Republic
}

\begin{abstract}
The paper deals with the application of Raman spectroscopy for milk fat determination. For a quality control of milk and dairy products the monitoring and analysis of milk nutritional composition is crucial. Commonly available milk was used for analyses in form of liquid milk samples and also dried milk droplets with fat concentration $0,1 \%-3,5 \%$. Raman spectroscopy brings benefits over traditional laboratory techniques in terms of effective, rapid and reagent free way of milk fat measurements. Acquired Raman spectra were assigned to appropriate milk elements focusing on milk fat components. Method accuracy for a content of fat in milk is discussed in paper and in comparison to results obtained by conventional Röse-Gottlieb gravimetric method and butyrometry show good agreement.
\end{abstract}

Keyword: Quality control; Fat content; Milk fat; Raman spectra; Spectroscopic analysis
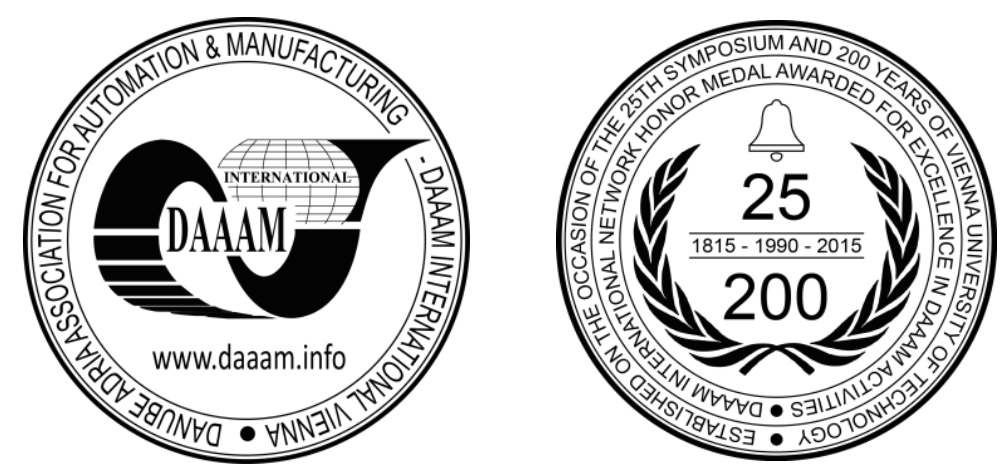

This Publication has to be referred as: Vaskova, H[ana] \& Buckova, M[artina] (2016). Spectroscopic Measurement and Analysis of Fat in Mi, Proceedings of the 26th DAAAM International Symposium, pp.0365-0370, B. Katalinic (Ed.), Published by DAAAM International, ISBN 978-3-902734-07-5, ISSN 1726-9679, Vienna, Austria DOI:10.2507/26th.daaam.proceedings.049 


\section{Introduction}

Milk fat assessment is necessary both in terms of food technology and in terms of maintaining the nutritional value. Milk and dairy products play not insignificant role in the human diet mainly for children. Milk and many kinds of dairy products serve as calcium, protein and vitamins A, D, E source. Main components of milk comprise milk proteins, carbohydrates and fat. The reliable information about these main components of milk is crucial characteristic for adequate technology application. Additionally, this information has to be labelled on milk or dairy products to inform customers about their nutritional parameters. Nowadays many people are looking for milk and dairy products with special nutritional properties like lower fat content, lactose-free or vitamin D fortified products. That is why dairy industry has to adjust milk to these special consumers' demands.

Bovine milk is composed of approximately $87 \%$ water, $4-5 \%$ lactose, $3 \%$ protein, $3-4 \%$ fat, $0.8 \%$ minerals and $0.1 \%$ vitamins. Amount and composition of milk fat is responsible for physical and sensory properties of dairy products. Knowledge of the fatty acids composition in milk is important, because of their influence on cardiovascular health. The main negative concern has been related to saturated fat content representing approximately $70 \%$ of total milk fat, only $30 \%$ of fat share is comprises unsaturated fatty acids [1]. Raman spectroscopic evaluation of fatty acids contained in vegetable oils can be found e.g. in [2], [3].

Especially the producers necessitate effective quality monitoring of raw milk composition and other ingredients as well as monitoring of quality confirmation of final dairy products. Common methods are based mostly on traditional laboratory techniques using usually sample pretreatment, application of many chemicals, several steps and time consuming analyses, skilled and experienced personnel. The principally used methods include the Rose-Gottlieb method and Gerber method, often named "butyrometric method". Both are time consuming and requiring chemicals and special lab equipment. Therefore, methods allowing fast, simple, ideally nondestructive and accurate analysis need to be developed and improved. From this point of view, new spectroscopic methods, e.g. ultraviolet-visible, infrared, luminescence or Raman spectroscopy seems to be promising techniques. Moreover, multivariate chemometric tools coupled with spectrometric methods can significantly increase their application (not only) in food analysis and may have value for solving problems in dairy science and technology [4, 5].

Applicability of Raman spectroscopy related to analysis of milk and dairy product has an increasing trend lately. In contrast to many other spectroscopic methods, Raman spectroscopy does not require optical purity of the samples and brings benefits of rapid, non-contact and nondestructive measurements. Raman spectroscopy, therefore represent a suitable tool for direct in situ analysis of the major constituents of food systems [6].

Determination of protein, carbohydrate and fat content and also their change during technology or storage conditions belong to the main topics studied in dairy science [6] - [9]. The most known incident in food chemistry and forensic toxicology was the melamin adulteration causa. In this case, Raman spectroscopy provided a very rapid screening test for melamine-adulterated dried milk [7, 10]. Additionally a portable compact Raman spectrometric system suitable for on-line analysis was constructed to determine melamine adulteration in milk powder [7]. Focused on milk fat, analysis of Raman spectra in combination with chemometrics methods was used to detect, classify and quantify the adulteration of butter with margarine [11]. The main objective of presented paper was to perform Raman spectroscopy for milk fat measuring and to study the essential features as method sensitivity and accuracy in comparison to conventional laboratory techniques.

\section{Experimental part}

\subsection{Materials}

To study the content of milk fat seven mixtures of commonly sold skimmed $(0,1 \%)$, semi-skimmed $(1,5 \%)$ and whole milk $(3,5 \%)$ were prepared and used for measurements. The concentrations of fat in milk were $0,1 \%, 0,8 \%$,

\begin{tabular}{llc}
\hline Calculated [\%] & Measured - Röse Gottlieb [\%] & Measured - Gerber method [\%] \\
\hline 0,1 & $0,074 \pm 0,010$ & - \\
0,8 & $0,872 \pm 0,031$ & $0,83 \pm 0,05$ \\
1,5 & $1,539 \pm 0,009$ & $1,55 \pm 0,05$ \\
2 & $2,015 \pm 0,029$ & $2,08 \pm 0,05$ \\
2,5 & $2,558 \pm 0,016$ & $2,63 \pm 0,11$ \\
3 & $3,048 \pm 0,037$ & $3,07 \pm 0,06$ \\
3,5 & $3,506 \pm 0,002$ & $3,60 \pm 0,08$ \\
\hline
\end{tabular}

Table 1 Fat content in milk mixtures.

$1,5 \%, 2,0 \%, 2,5 \%, 3,0 \%$ and 3,5\%. Röse-Gottlieb gravimetric method as well as Gerber method was used for verification of calculated fat concentrations. Results are listed in Table 1. In order to eliminate influence of proteins, all 
samples have constant value of protein. The Milk samples were measured in two forms: directly in liquid form in open aluminum dishes at a laboratory temperature and in form of dried milk droplets on aluminum plates.

\subsection{Raman spectroscopy}

Raman spectroscopy as the vibrational spectroscopic method provides a specific chemical fingerprint of every single chemical substance and its modifications in the form of Raman spectra. Vibrations of particular molecular bonds cause a slight characteristic changes in wavelengths in scattered photons. These wavelength shifts then facilitate the material identification and structural assessments. Substantial benefits arise from many advantages of the method: Raman spectroscopy is relatively rapid, non-destructive, contactless, applicable to all states of matter of the in different forms without special requirements for sample preparation, independent on chemicals, usable as in situ analysis, usable for measuring through transparent glass or polymeric covering layers. Since the Raman scattering is a weak effect, some adverse effects can influence the quality of spectral response. Luminescence, for instance, as much stronger quantum effect can overlap Raman spectra with its intensity and mask spectral information.

Raman spectroscopy finds more and more applications across scientific areas such as chemistry, biochemistry, material science, mineralogy, arts, medicine; method is used for pharmaceutical, forensic and security purposes and recently begins to penetrate also to food industry $[12,13]$.

Raman spectra of all samples were measured on Renishaw InVia Basis Raman microscope using NIR diode laser $(785 \mathrm{~nm}$ ) with maximum output power $300 \mathrm{~mW}$. Leica DM 2500 confocal microscope with the resolution $2 \mu \mathrm{m}$ was coupled to the Raman spectrometer. All measurements were collected with $10 \mathrm{~s}$ exposure time and 3 accumulations. The samples were firstly scanned in range 100 to $3200 \mathrm{~cm}-1$ with $2 \mathrm{~cm}-1$ spectral resolution. After determining the principle peaks the spectral range was reduced approximately to the area $300-1800 \mathrm{~cm}^{-1}$.

2.3. Conventional methods for milk fat analysis

Röse-Gottlieb method is based on extraction using a mixture of organic solvents and gravimetric determination of milk fat expressed as $\mathrm{g}$ of extracted fat per $100 \mathrm{~g}$ of milk. Before extraction, all milk samples are heated to $38 \pm 1^{\circ} \mathrm{C}$ to ensure complete homogenization. $100 \mathrm{ml}$ milk samples are digested by NH3 solution $(25 \% \mathrm{v} / \mathrm{v})$ and mixed with ethanol $(96 \%$ $\mathrm{v} / \mathrm{v})$. The extraction is performed 3 times using the mixture of diethyl ether and petroleum ether (1:1). Finally the solvent phase is evaporated under vacuum and fat is weighted and calculated. This method is based on European Standard EN ISO 1211 and it is considered as reference method for milk fat determination [14].

Gerber method, called also butyrometry is often used in laboratories because it is relative simple, fast, low-cost and suitable for a quite high sample throughput. On the other hand, highly concentrated sulphuric acid is used, what involves a certain risk and potential environmental damage. Moreover, handling the butyrometer requires practical skills [15]. Procedure: $10 \mathrm{ml}$ of Gerber sulfuric acid is placed into butyrometer tube, $11 \mathrm{ml}$ of well homogenized milk sample and $1 \mathrm{ml}$ of amylalcohol is added. Butyrometric tube is locked, well shaken and centrifuged. The fat level is obtained from butyrometer scale under temperature $65^{\circ} \mathrm{C}$. Whole procedure is carried out according to [16]. This method usually is used as a screening test.

\section{Results}

In the first instance the spectroscopic measurements were performed on milk samples with $0,1 \%-3,5 \%$ fat content. Due to the appearing luminescence, dried milk droplets were used for measuring and considered for evaluation. Essential bands for milk components are listed in Table 2. Obtained Raman spectra are shown in Fig. 1A. Raman spectra of all droplets samples are displayed in Fig. 2.

\begin{tabular}{ll}
\hline Raman peak $\left[\mathrm{cm}^{-1}\right]$ & Assignment to vibrations of chemical bonds \\
\hline 1005 & Phenylalanine ring breathing \\
1008 & $\mathrm{C}-\mathrm{C}$ stretch of carotenoids \\
1150 & $\mathrm{C}-\mathrm{CH}_{3}$ rocking of carotenoids \\
1267 & $=\mathrm{C}-\mathrm{H}$ symmetric rocking \\
1303 & $\mathrm{CH}_{2}$ in-plane twist \\
1443 & $\mathrm{CH}_{2}$ scissoring \\
1525 & $\mathrm{C}=\mathrm{C}$ stretching of carotenoids \\
1658 & $\mathrm{C}=\mathrm{C}$ cis double bond stretching \\
1748 & $\mathrm{C}=\mathrm{O}$ ester-carbonyl stretching \\
\hline
\end{tabular}

Table 2 Raman bands and their assignments. 

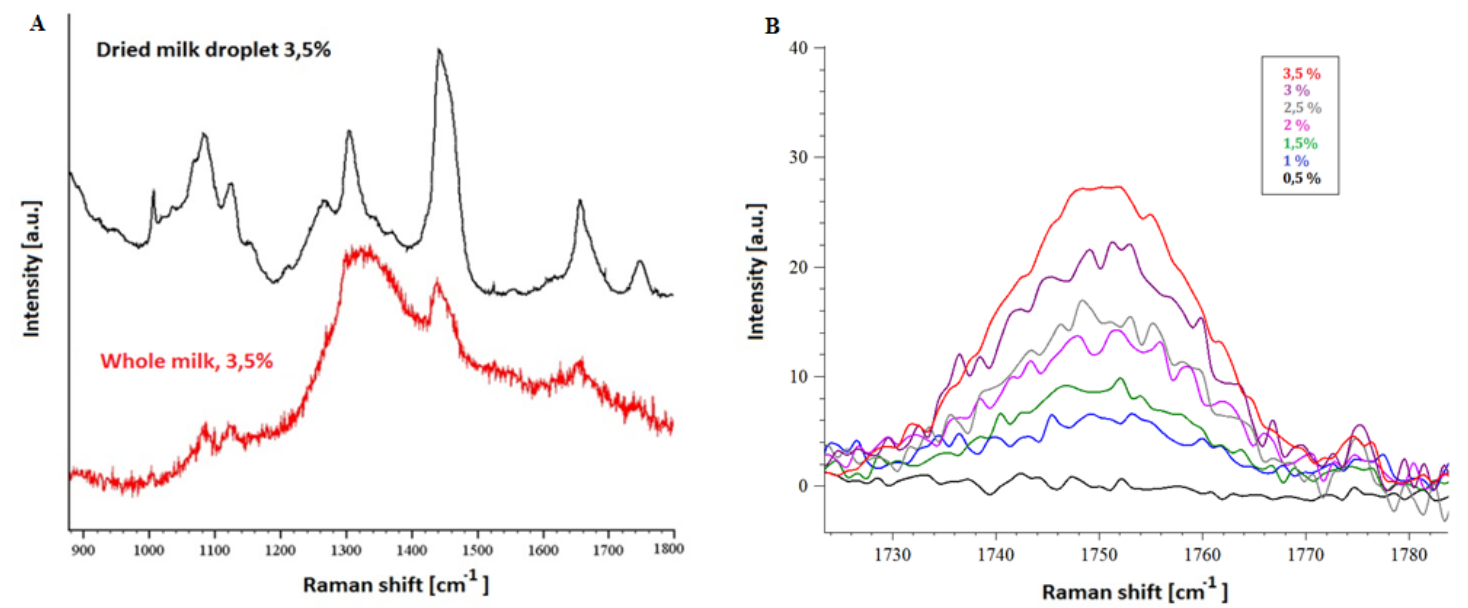

Fig. 1. A - Raman spectra of dried milk droplet and liquid milk, B - Raman spectra of dried milk droplets the increase of the normalized intensity at $1748 \mathrm{~cm}-1$ with the content of fat

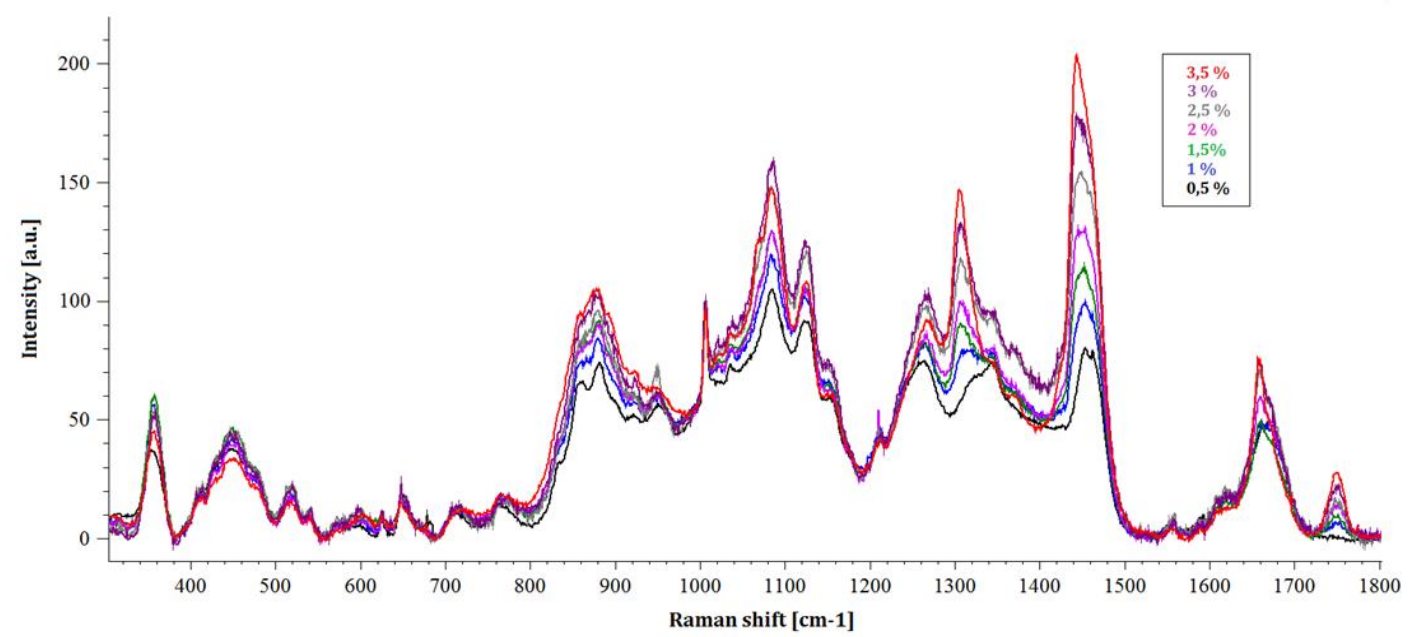

Fig. 2. Raman spectra of dried milk droplets with fat concentration $0,1 \%-3,5 \%$

Raman spectroscopy
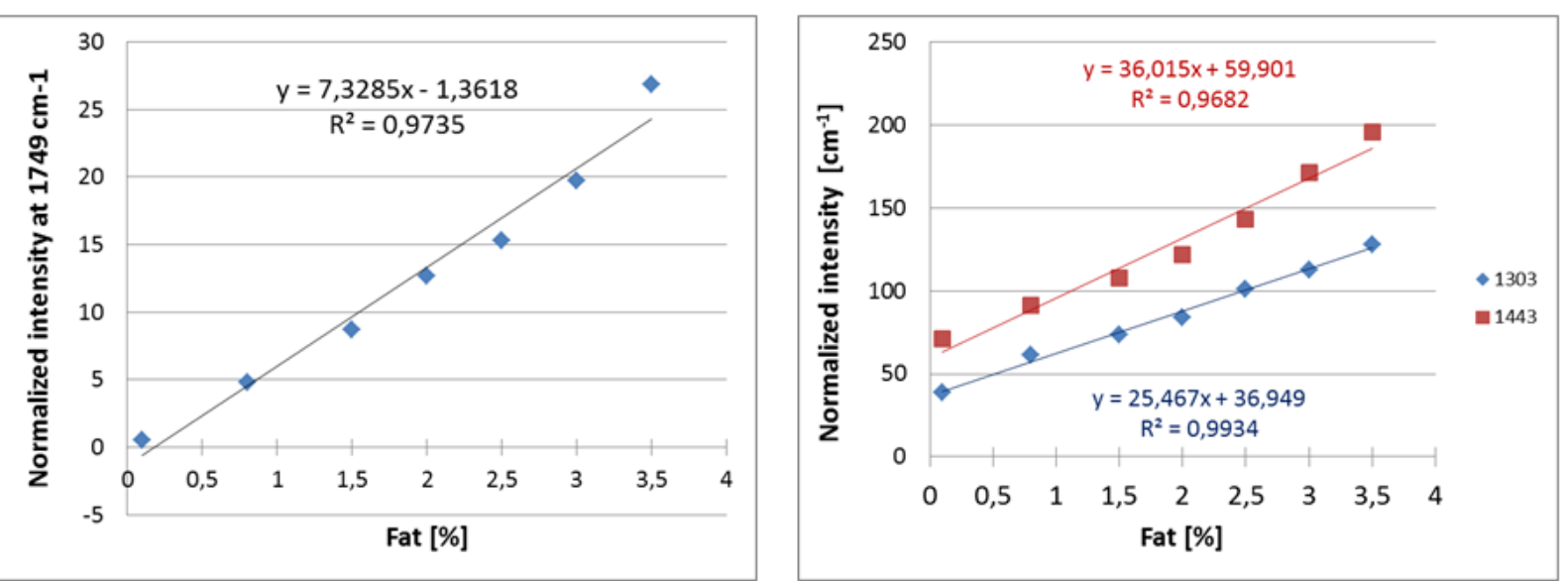

Fig. 3. Dependence of the normalized intensity at $1749 \mathrm{~cm}-1,1303 \mathrm{~cm}-1$, and $1443 \mathrm{~cm}-1$ on the fat content in dried milk droplets 

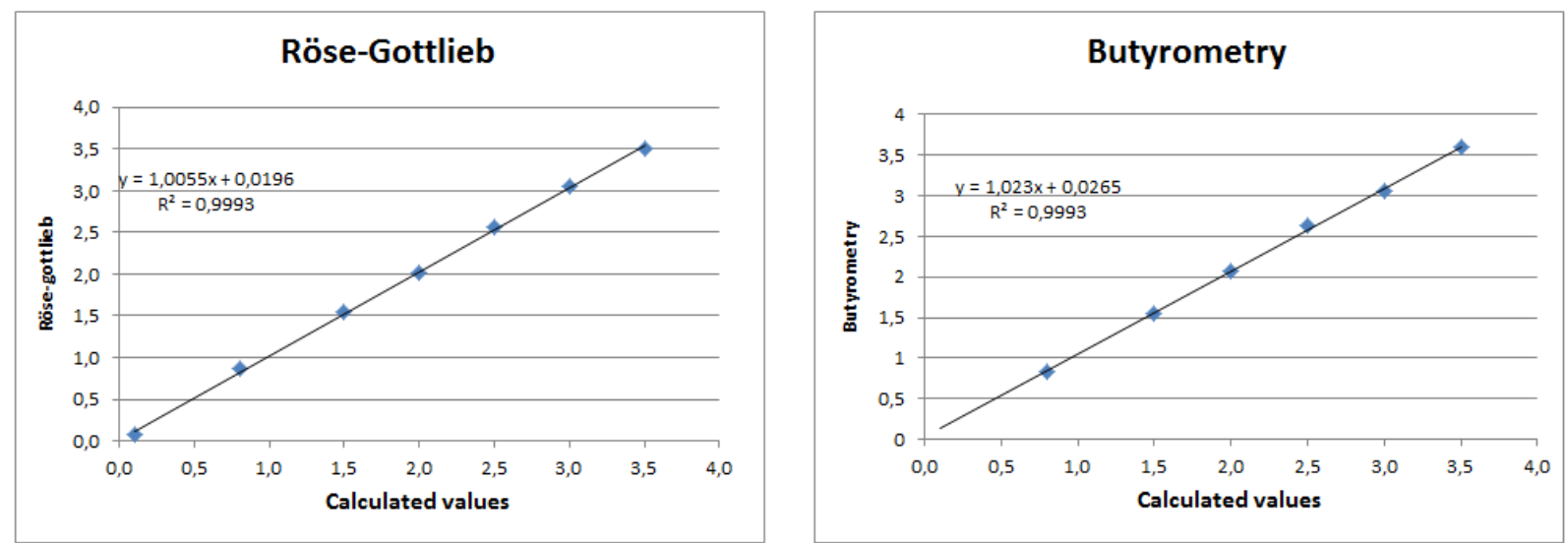

Fig. 4. Measured vs. calculated values for Röse-Gottlieb and butyrometric method.

Milk fat in Raman spectra is represented by $\mathrm{C}=\mathrm{O}$ stretching of the ester groups of triglycerides at $1748 \mathrm{~cm}^{-1}$, whereas the $1005 \mathrm{~cm}^{-1}$ phenylalanine ring breathing band is indicative of protein [4]. Assuming the protein content does not alter, this peak is taken as a standard to normalise intensity values. The $\mathrm{CH}_{2}$ deformation vibrations at $1303 \mathrm{~cm}^{-1}$ and $1443 \mathrm{~cm}^{-1}$ are specific to the saturated fatty acids, $\mathrm{C}=\mathrm{C}$ at $1654 \mathrm{~cm}^{-1}$ for unsaturated fatty acids in cis configuration [9].

For the evaluation of fat content in samples, the attention was directed to three significant bands: $1303 \mathrm{~cm}^{-1}$, $1443 \mathrm{~cm}^{-1}$ and $1748 \mathrm{~cm}^{-1}$. The baseline correction was applied on all spectra and the spectra were normalized. Details of the spectral response for band 1748 are displayed in Fig. 1B.

The linear dependence of the normalized intensities was revealed for all three examined bands and are shown in Fig. 3. In all cases the linearity exhibit quite high accuracy. Therefore based on a set of calibration data and specified procedure of data processing it is possible to determine the amount of fat in the samples. More proper for the evaluation and data processing seems to be the band $1748 \mathrm{~cm}^{-1}$ due to its solitary position in the spectra. However the other bands could be used for the confirmation. The accuracies for Röse-Gottlieb and butyrometry are shown in Fig. 4. In comparison with the conventional methods Raman spectroscopy as an innovative method for this application shows a very good agreement.

\section{Conclusion}

Raman spectroscopy was used as an innovative method for measuring the fat contained in milk. Acquired data indicate that to obtain more precise spectral response, because of the appearing luminescence masking the Raman signal, it is more proper to perform the measurements on dried milk droplets instead of liquid milk samples. Results acquired in this study show that on the basis of characteristic bands for saturated fatty acids it is possible to distinguish different fat concentrations in milk. Raman spectroscopic evaluation brings advantages over traditional methods mainly in sense of simplicity, rapidity and no use of chemical reagents with the only need to prepare the milk droplets. These aspects of measuring mean costs and time savings and according to well comparable results in accuracy Raman spectroscopy seems to be promising method to enlarge the range of conventional laboratory techniques for milk fat identification.

\section{Acknowledgements}

This work is supported by the Ministry of Education, Youth and Sports of the Czech Republic, by European Regional Development Fund under the project CEBIA-Tech No. CZ.1.05/2.1.00/03.0089 and its continuing project, i.e. by Narodni program udrzitelnosti No. MSMT-7778/2014

\section{References}

[1] P. C. Pereira, "Milk nutritional composition and its role in human health," Nutrition, vol. 30, pp. 619-627, 2014.

[2] M. Buckova, H. Vaskova, M. Tobolova and V. Kresalek, "Spectroscopic Screening of Degradation Process in Edible Oils and Its Mathematical Evaluation,“ International Journal of Mathematical Models and Methods in Applied Sciences [online], vol. 8, pp. 319-328, 2014.

[3] B. Muik, B. Lendl, A. Molina-Diaz and M. J. Ayora-Canada, "Direct monitoring of lipid oxidation in edible oils by Fourier transform Raman spectroscopy," Chemistry and Physics of Lipids, vol. 134, pp. 173-182, 2005.

[4] C. M. McGoverin, A. S. S. Clark, S. E. Holroyd and K. C. Gordon, "Raman spectroscopic quantification of milk powder constituents," Analytica Chimica Acta, vol. 673, pp. 26-32, 2010. 
[5] M. A. P. Rodriguez, J. Petrini, E. M. Ferreira, L. R. M. B. Mourao, M. Salvian, L. D. Cassoli, A. V. Pires, P. F. Machado and G. B. Mourao, "Concordance analysis between estimation methods of milk fatty acid content," Food Chemistry, vol. 156, pp. 170-175, 2014.

[6] E. C. Y. Li-Chan, "The applications of Raman spectroscopy in food science," Trends in Food Science and Technology, vol. 7, pp. 361-370, 1996.

[7] D. Yang and Y. Ying, "Applications of Raman Spectroscopy in Agricultural Products and Food Analysis: A Review,” Applied Spectroscopy Reviews, vol. 46, pp. 539-560, 2011.

[8] J. Fontecha, J. Bellanato and M. Juarez, "Infrared and Raman Spectroscopic Study of Casein in Cheese: Effect of Freezing and Frozen Storage," Journal of Dairy Science, vol. 76, pp. 3303-3309, 1993.

[9] S. Gallier, K. C. Gordon and H. Singh, "Chemical and structural characterisation of almond oil bodies and bovine milk fat globules," Food Chemistry, vol. 132, pp. 1996-2006, 2012.

[10] E. Domingo, A. A. Tirelli, C. A. Nunes, M. C. Guerreiro and S. M. Pinto, "Melamine detection in milk using vibrational spectroscopy and chemometrics analysis: A review," Food Research International, vol. 60, pp. 131139,2014

[11] R. S. Uysal, I. H. Boyaci, H. E. Genis and U. Tamer, "Determination of butter adulteration with margarine using Raman spectroscopy," Food Chemistry, vol. 141, pp. 4397-4403, 2013.

[12] J. M. Chalmers, G. E. Howell and M. D. Hargreaves, Infrared and Raman spectroscopy in forensic science. 1st pub. Chichester, West Sussex, UK: Wiley, 2012

[13] N. B. Colthup, L. H. Daly and S. E. Wiberley, Introduction to infrared and Raman spectroscopy. 3rd ed. San Diego: Academic Press, 1990.

[14] Milk - Determination of fat content - Gravimetric Method (Reference method), European Standard EN ISO 1211:2010.

[15] R. Badertscher, T. Berger and R. Kuhn, "Densitometric determination of the fat content of milk and milk products," International Dairy Journal, vol. 17, pp. 20-23, 2007.

[16] IDF 105:2008 Milk - Determination of fat content - Gerber butyrometers 